\title{
SN-series Pseudospark Switches, Operating Completely without Permanent Heating. New Prospects of Application in Pulsed Power
}

\author{
V.D. Bochkov*, D.V. Bochkov, V.M. Dyagilev and V.G. Ushich \\ Pulsed Technologies Ltd., Yablochkova 5, Ryazan, Russia
}

\begin{abstract}
Since 1990s the process of exclusion of gas-discharge and vacuum switches (GDSs and VSs) from pulsed power systems has been conditioned by two major factors - insufficient service time and necessity to supply filament circuits of GDSs and VSs. In the report, design and long-term test results of pseudospark switches (PSSs) are presented. The PSSs are to a great extent free of the mentioned shortcomings; in particular, the switches are capable of operating without filament, providing a wide variety of perspective applications of the tubes in pulsed power.
\end{abstract}

PACS numbers: 52.75.-d, 52.77.Fv, 84.32.Dd, 52.75.Kq

\section{Introduction}

Hydrogen thyratrons as well as spark gaps were the first and till the end of last millennium the most widespread switching elements in pulsed power. Their physical and technical parameters allow them to be capable of operating in systems where a totality of parameters such as high timing stability, independence of anode voltage, high pulse repetition rates, resistance to the change in environmental conditions (including high radiation doze), surge capability, ability to switch oscillating discharge current, offering high rates of current rise up to $10^{11} \mathrm{~A} / \mathrm{s}$, etc. is required.

Meanwhile the presence of heated parts - cathode and hydrogen reservoir, supplied from external sources, defines a number of shortcomings of hydrogen thyratrons if compared to competitors - spark and vacuum switches, ignitrons, solid state switches. Among the drawbacks there are relatively short service times and weak durability of hot cathode, containing alkaline-earth metals. Dispersion and subsequent activation of surfaces of high-voltage electrodes by the elements with low work function leads to reduction of hold-off voltage in the device, whereas high temperature of cathode reduces discharge development time nearby. Heated parts make application circuits more complicated, especially when cathode and anode are under high voltage; reduce reliability and service time, increasing readiness time.

Designed and built in the end of 1980s so-called pseudospark switches (PSSs) [1-4] do not have hot cathode, however to maintain a stable working gas (hydrogen, deu-

* corresponding author; e-mail: pulsetech@mail.ru terium) pressure the switches require a constant heating of hydrogen reservoir. The presence of the reservoir does not allow to use the potential of PSSs completely.

In Russia designed and serially produced are thyratrons of 2 types: TDI- and TPI-type. TDI-type thyratrons have low inductivity and are capable of conducting currents up to $200 \mathrm{kA}$ at current rise rates up to $5 \times 10^{12} \mathrm{~A} / \mathrm{s}$, anode delay time 50-200 ns and time jitter of less than 4 ns $[5,6]$. TPI-type thyratrons are intended for switching up to tens of $\mathrm{kA}$ with discharge development time up to $1-5 \mathrm{~ns}$, time jitter $0.4-5 \mathrm{~ns}$, and recovery time less than $1 \mu \mathrm{s}[8,9]$. By using a specially designed built-in device of SRNV (Russian abbreviation of Selective Reversible Hydrogen Leakage) a long service time of the switches has been achieved without application of external filaments power supplies.

\section{Experimental results}

The initial construction of SRNV was built and tested in 1988 in high-power gas discharge switch with cold cathode - trioplasmatron. The device SRNV-1 (Fig. 1) comprised two basic parts - a cavity with heated reservoir (HR), in which a reserve of hydrogen in titanium hydride was kept, and hermetically sealed metal selective membrane (SM), dividing operating volume of the switch from the reservoir. The membrane in cold state is impermeable for all gases, but after warming up it leaks hydrogen only in both directions - out of reservoir as well as out of filling volume. The direction of hydrogen transition is from the volume with high partial pressure (PP) to the volume with lower PP. In order to supply hydrogen into the thyratron volume heating voltage is applied to hydrogen reservoir and titanium hydride releasing hydrogen maintains necessary pressure inside the 


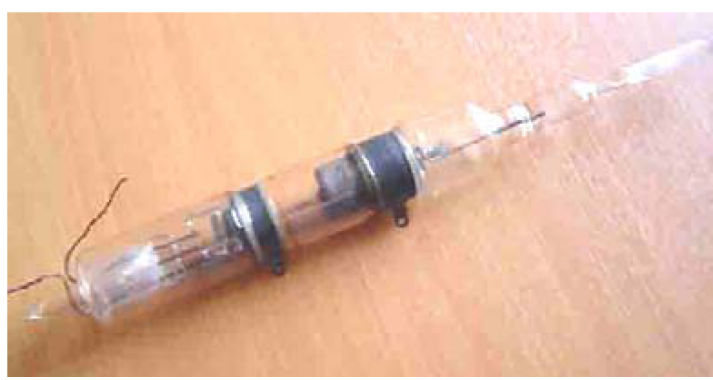

Fig. 1. Metal-glass construction of SRNV-1.

switch. The warmed selective membrane leaks hydrogen due to overpressure diffusion.

In the designation of SRNV the essence of one of the device features is placed - reversibility, i.e. capability both to replenish hydrogen in the thyratron volume and to return it into a hydrogen reservoir, e.g. in case of accidental overdose of working gas.

The tests have shown that the device is capable of operating without heating voltage application within more than 1 year.

Another design of SRNV-3 was intended for maintaining a stable pressure of high purity hydrogen in devices, operating with additives of hydrogen in aggressive gases environments (metal-vapor lasers, metal halide and HF lasers, etc.) and as a future prospect in the hydrogen fuel elements. The tests of sealed-off $\mathrm{CuBr}^{+} \mathrm{Ne}^{+} \mathrm{H}_{2}$ lasers at the Atmosphere Optics Institute SB RAS (Tomsk, Russia) have shown that application of 2 additives using SRNV-3 increases average radiation power twice and even fivefold depending on gas-discharge tube diameter [10]. Besides, the hydrogen additive allows increasing maximum pump frequency up to $250 \mathrm{kHz}$. At that in a laser tube having $2.6 \mathrm{~cm}$ in diameter and $76 \mathrm{~cm}$ in length, radiation power was $1.5 \mathrm{~W}$, at frequency $200 \mathrm{kHz}-3 \mathrm{~W}$ and at frequency $100 \mathrm{kHz}-10.5 \mathrm{~W}$.

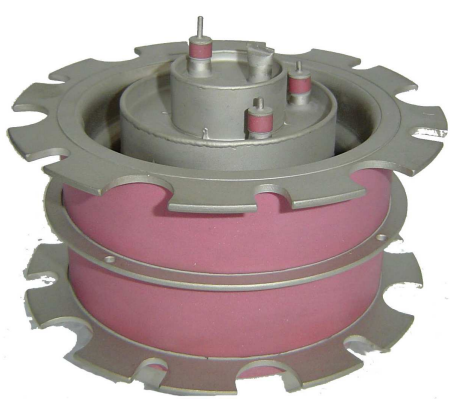

Fig. 2. TDI1-150k/25SN thyratron with metal-ceramic SRNV-6 device.

Especially for thyratrons we have designed and fabricated compact devices SRNV-6 and SRNV-7. Pulsed Technologies Ltd. has "know-how" of production and application of the devices. The presence of SRNV in TDI-SN and TPI-SN thyratrons not only does not make

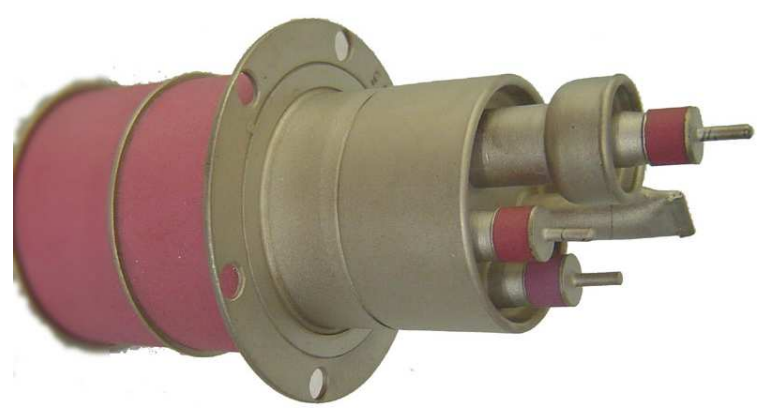

Fig. 3. TDI1-50k/16SN thyratron with metal-ceramic built-in SRNV-7.

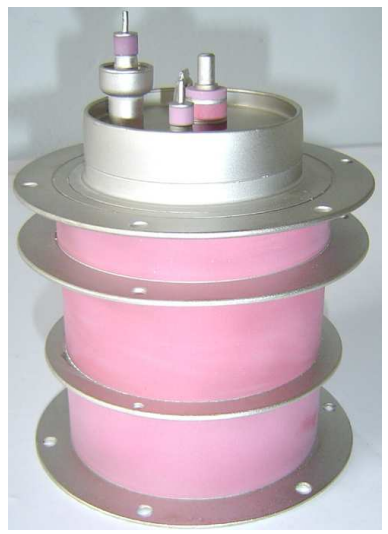

Fig. 4. TPI1-10k/50SN thyratron with metal-ceramic built-in SRNV-7.

harm to their basic electrical parameters in respect of a "common" line of TDI- and TPI-thyratrons. On the contrary, a constructive addition SRNV-6 and SRNV-7 mounted into a cathode assembly (Fig. 2-4) significantly improves thyratrons operation characteristics. It allows having at least 4 advantages over usual thyratrons.

The first advantage is the instant operation readiness, which means that the device requires neither fast (20-60 seconds) nor usual (5-15 minutes) heating, all known pulse hydrogen thyratrons are subject to.

The thyratron does not require permanent heating of hydrogen reservoir (HR). The device is capable of operating with switched-off heating, which simplifies thyratron application when operation with high cathode potential is required.

Due to separation of hydrogen reservoir from the operating volume of thyratron there is no reservoir poisoning by various gases, released by electrodes during operation, thus providing the thyratron parameters stability.

The device has the best parameters stability even if environment temperature is unstable. The switch is being delivered ready-to-use and does not need a heater voltage circuit. In the course of operation due to absorption of buffer gas a hydrogen pressure is being diminished. It can be noticed by increase of delay time (e.g. from $150 \mathrm{~ns}$ to $500 \mathrm{~ns})$. 
In order to recover gas pressure one needs to execute a routine maintenance procedure:

A high anode voltage must be switched off, whereas heater voltage must be supplied to hydrogen generator and to additional electrode - selective membrane. Approximately within 5-10 minutes a recovery occurs and the switch can be operated like a new one. These cycles can be repeated many times to provide for resource of the thyratron.

The thyratrons in modes with switched energy up to 5-7 kJ per shot, at anode voltages up to $35 \mathrm{kV}$, for oscillated discharge $\left(I_{2}=0.9 I_{1}\right.$, where $I_{1}$ is amplitude of the first semi-wave and $I_{2}$ is amplitude of the second current semi-wave), delivering currents up to $150 \mathrm{kA}$, are capable of operating for a rather long time (20000-30000 shots) without hydrogen reservoir heating, however in case of working gas regeneration - above 1 million pulses.

Presently, in Pulsed Technologies Ltd. a work on design and construction of principally novel device SRNV-8 with reduced dimensions, intended for no-hydrogen-reservoir-heating operation of compact thyratrons TDI1-50k/16, TPI1-0.2k/12, TPI1-1k/20, TPI1-1k/35 etc. is being conducted.

\section{Conclusions}

By construction of SN-thyratrons we have achieved a goal to have principally novel switches, combining the best features of thyratrons, vacuum and spark gaps, solid state switches. The solution dramatically improves operational characteristics of the switches, in particular, extends operating environmental temperature range, improves stability, simplifies circuitry when operation with high cathode (and/or anode) potential is required, allows to have instant readiness for operation, keeping all the best qualities of TDI- and TPI-thyratrons.

\section{Acknowledgments}

The work was supported by Pulsed Technologies Ltd. Co in the framework of the company's R\&D Programs.

\section{References}

[1] J. Christiansen, C. Schultheiss, Z. Phys. A 290, 35 (1979).

[2] G.F. Kirkman, M.A. Gundersen, Appl. Phys. Lett. 49, 494 (1986)
[3] V.D. Bochkov, S.Sh. Zaidman, D.P. Patrushev, Instruments and Experimental Techniques 5, 229 (1992) (in Russian).

[4] A. Kuthi, J. Liu, C. Young, L.C. Lee, M. Gundersen, Pseudospark Based Pulse Generator for Corona Assisted Combustion Experiments, ONR Review, August 5-7, 2002.

[5] V.D. Bochkov, V.M. Djagilev, V.G. Ushich, O.B. Frants, Yu.D. Korolev, I.A. Shemyakin, K. Frank, IEEE Trans. on Plasma Sci. 29, 802 (2001).

[6] N.V. Zharova, N.A. Ratakhin, A.V. Saushkin, V.F. Fedushchak, A.A. Erfort, Instruments and Experimental Techniques 49, 384 (2006).

[7] V.A. Gribkov, M. Scholz, V.D. Bochkov, A.V. Dubrovsky, R. Miklaszewski, L. Karpinski, S. Lee, P. Lee, Journal of Physics D: Appl. Phys. 37, 2107 (2004).

[8] O.V. Anchugov, Yu.G. Matveev, D.A. Shvedov, V.D. Bochkov, D.V. Bochkov, V.M. Dyagilev, V.G. Ushich, S.F. Mikhailov, V.G. Popov, 2007 PPPC, Albuquerque, USA, 2007.

[9] A.V. Akimov, P.V. Logachev, V.D. Bochkov, D.V. Bochkov, V.M. Dyagilev, V.G. Ushich, 2007 PPPC, Albuquerque, USA, 2007.

[10] D.V. Shiyanov, V.B. Sukhanov, O.S. Andrienko, G.S. Evtushenko, V.D. Bochkov, Proc. of Sixth International Conference on Atomic and Molecular Pulsed Lasers (AMPL-2003), Tomsk, 2003, Russia.

[11] V.D. Bochkov, V.M. Dyagilev, Yu.D. Korolev, V.G. Ushich, Instruments and Experimental Techniques 41, 676 (1998).

[12] V.D. Bochkov, V.M. Dyagilev, V.G. Ushich, O.B. Frants, Yu.D. Korolev, I.A. Shemyakin, K. Frank, IEEE Trans. Plasma Sci. 29, 802 (2001).

[13] V.D. Bochkov, D.V. Bochkov, V.M. Dyagilev, V.N. Kudinov, V.G. Ushich, V.A. Glouschenkov, R.Yu. Yusupov, Proc. 2002 International Power Modulator Conference, 475 (2002).

[14] V.A. Gribkov, M. Scholz, V.D. Bochkov, A.V. Dubrovsky, R. Miklaszewski, L. Karpinski, S. Lee, P. Lee, J. Phys. D: Appl. Phys. 37, 2107 (2004).

[15] V.D. Bochkov, Yu.D. Korolev, in: Encyclopedia of Low Temperature Plasma, Ed. V.E. Fortov, Nauka Publishing, Moscow 2000, p. 446 (in Russian). 